\title{
Approximation of Asymptotic Expansion of Wavelets
}

\author{
Shyam Lal \\ Banaras Hindu University \\ Institute of Science \\ Department of Mathematics \\ Varanasi-221005, India
}

\begin{abstract}
In this paper, the approximation of asymptotic expansion for the wavelet is obtained and the approximation of asymptotic expansions for generalized Mexican hat wavelet and the wavelet corresponding to $m^{\text {th }}$ order cardinal $B$-spline are obtained.

Estimates of present investigations are based on Wong's method.
\end{abstract}

\section{General Terms}

Wavelet, Approximation, Basic wavelet

\section{Keywords}

First order cardinal $B-$ spline, asymptotic approximation, $m^{\text {th }}$ order cardinal $B$-spline, asymptotic expansion, wavelet

transform, generalized mexican hat wavelet, Mellin transform

\section{INTRODUCTION}

At first in 1989, Wong [1] studied the asymptotic approximation of certain integrals. Wavelet approximation plays an important role in Mathematics, Computer science and Technology . Approximation of asymptotic expansion of wavelet has been studied by Sweldens and Piessens [2],Pathak and Pathak [3], Wong [4] and Ashurov and Butaev [5] etc. But till now no work seems to have been done to obtain the approximation of asymptotic expansion of generalized Mexican hat wavelet and wavelet corresponding to $m^{\text {th }}$ order cardinal B-spline. In an attempt to make an advance study in this direction, in this paper, the estimates for asymptotic expansion of generalized Mexican hat wavelet and $m^{t h}$ order cardinal B-spline wavelets are determined. These estimates are new, better and sharper than all previously known estimates.

\section{DEFINITIONS}

If a function $\psi \in L^{2}(R)$ satisfies the "admissibility" condition:

$$
C_{\psi}=: \int_{-\infty}^{\infty} \frac{|\hat{\psi}(\omega)|^{2}}{|\omega|} d \omega<\infty
$$

then $\psi$ is called a "basic wavelet". Relative to every basic wavelet $\psi$, the integral wavelet transform (IWT) of a function $f \in L^{2}(R)$

\author{
Susheel Kumar \\ Govt.P.G.College, Joshimath \\ Faculty of Science \\ Department of Mathematics \\ Chamoli Uttarakhand-246443, India
}

is defined by

$$
\left(W_{\psi} f\right)(b, a)=:|a|^{-\frac{1}{2}} \int_{-\infty}^{\infty} f(t) \overline{\psi\left(\frac{t-b}{a}\right)} d t
$$

where $a, b \in R$ with $a \neq 0$, (Chui [6]).

By setting

$$
\psi_{b ; a}=:|a|^{-\frac{1}{2}} \psi\left(\frac{t-b}{a}\right)
$$

the IWT defined in 2) can be written as

$$
\left(W_{\psi} f\right)(b, a)=\left\langle f, \psi_{b ; a}\right\rangle .
$$

Using Parsewall identity, it can also be written as

$$
\begin{aligned}
\left(W_{\psi} f\right)(b, a) & =\frac{1}{2 \pi}\left\langle\hat{f}, \hat{\psi}_{b ; a}\right\rangle \\
& =\frac{a|a|^{-\frac{1}{2}}}{2 \pi} \int_{-\infty}^{\infty} \hat{f}(\omega) \overline{e^{-i b \omega} \hat{\psi}(a \omega)} d \omega \\
& =\frac{a|a|^{-\frac{1}{2}}}{2 \pi} \int_{-\infty}^{\infty} \hat{f}(\omega) e^{i b \omega} \overline{\hat{\psi}}(a \omega) d \omega,
\end{aligned}
$$

Approximation of asymptotic expansion for the general integral

$$
I(x)=\int_{0}^{\infty} F(t) h(x t) d t
$$

was discussed by Wong [1]. Considering the basic idea of Wong [1] related to approximation of asymptotic expansion of general integral, the derivation for the approximation of asymptotic expansion of a wavelet may be obtained.

Suppose that $F(t)$ has an asymptotic expansion as

$$
F(t) \sim \sum_{k=0}^{n-1} c_{k} t^{k+\sigma-1}+F_{n}(t), \text { as } t \rightarrow 0^{+},
$$

where $0<\sigma \leq 1, F_{n}(t)=\sum_{k=n}^{\infty} c_{k} t^{k+\sigma-1}$. 
The generalized Mellin transform of $h$, denoted by $M[h ; z]$, is defined by

$$
M[h ; z]=\lim _{\epsilon \rightarrow 0^{+}} \int_{0}^{\infty} t^{z-1} h(t) e^{-\epsilon t^{p}} d t .
$$

By equation (7) and [Wong [1], p.216],

$$
I(x)=\sum_{k=0}^{n-1} c_{k} M[h ; k+\sigma] x^{-(k+\sigma)}+\delta_{n}(x),
$$

where approximation or error bound $\delta_{n}$ is given by

$$
\delta_{n}(x)=\lim _{\epsilon \rightarrow 0^{+}} \int_{0}^{\infty} F_{n}(t) h(x t) e^{-\epsilon t^{p}} d t .
$$

The condition for satisfying the result 9 is already given by Wong ([1], theorem 6, p.217).

\section{APPROXIMATION OF ASYMPTOTIC EXPANSION FOR A WAVELET}

The approximation of asymptotic expansion of $\left(W_{\psi} f\right)(b, a)$ for fixed $b$ and $|a|$, has been derived. Estimates of present investigations are based on Wong's method.

$$
\begin{aligned}
\left(W_{\psi} f\right)(b, a) & =\frac{a|a|^{-\frac{1}{2}}}{2 \pi} \int_{-\infty}^{\infty} \hat{f}(\omega) e^{i b \omega} \overline{\hat{\psi}}(a \omega) d \omega \\
& =\frac{a|a|^{-\frac{1}{2}}}{2 \pi} \int_{0}^{\infty} \hat{f}(\omega) e^{i b \omega} \overline{\hat{\psi}}(a \omega) d \omega \\
& +\frac{a|a|^{-\frac{1}{2}}}{2 \pi} \int_{0}^{\infty} \hat{f}(-\omega) e^{-i b \omega} \overline{\hat{\psi}}(-a \omega) d \omega
\end{aligned}
$$

Assume that $\hat{f}(\omega)$ has expansion of the form

$$
\hat{f}(\omega)=\sum_{k=0}^{\infty} c_{k} \omega^{k+\sigma-1} \text { as } \omega \rightarrow 0^{+},
$$

where $0<\sigma \leq 1$.

Next,

$$
\begin{aligned}
F(\omega) & =\hat{f}(\omega) e^{i b \omega} \\
& =\left(\sum_{k=0}^{\infty} c_{k} \omega^{k+\sigma-1}\right)\left(\sum_{r=0}^{\infty} \frac{(i b \omega)^{r}}{r !}\right) \\
& =\sum_{k=0}^{\infty} d_{k} \omega^{k+\sigma-1} ; \text { as } \omega \rightarrow 0^{+} \\
& =\sum_{k=0}^{n-1} d_{k} \omega^{k+\sigma-1}+F_{n}(\omega) \text { as } \omega \rightarrow 0^{+},
\end{aligned}
$$

where

$$
F_{n}(\omega)=\sum_{k=n}^{\infty} d_{k} \omega^{k+\sigma-1}
$$

and

$$
d_{k}=\sum_{r=0}^{k} \frac{(i b)^{r}}{r !} c_{k-r}
$$

Further assuming that

$$
\overline{\hat{\psi}}(\omega) \sim e^{i \tau \omega^{p}} \sum_{r=0}^{\infty} b_{r} \omega^{-r-\alpha} ; \alpha>0, p \geq 1, \omega \rightarrow+\infty, \tau \neq 0
$$

and

$$
\overline{\hat{\psi}}(\omega)=O\left(\omega^{\rho}\right) ; \omega \rightarrow 0^{+}, \rho+\sigma>0 .
$$

By equations 9 and 11 ,

$\int_{0}^{\infty} e^{i b \omega} \hat{f}(\omega) \overline{\hat{\psi}}(a \omega) d \omega=\sum_{k=0}^{n-1} d_{k} M[\overline{\hat{\psi}}(\omega) ; k+\sigma] a^{-k-\sigma}+\delta_{n}^{(1)}(a)$,

where

$$
\delta_{n}^{(1)}(a)=\lim _{\epsilon \rightarrow 0^{+}} \int_{0}^{\infty} F_{n}(\omega) \overline{\hat{\psi}}(a \omega) e^{-\epsilon \omega} d \omega .
$$

Similarly,

$$
\begin{aligned}
\int_{0}^{\infty} e^{-i b \omega} \hat{f}(-\omega) \overline{\hat{\psi}}(-a \omega) d \omega= & \sum_{k=0}^{n-1} d_{k}(-1)^{k+\sigma+1} \\
& M[\overline{\hat{\psi}}(\omega) ; k+\sigma] a^{-k-\sigma}+\delta_{n}^{(2)}(a),
\end{aligned}
$$

where

$$
\delta_{n}^{(2)}(a)=\lim _{\epsilon \rightarrow 0^{+}} \int_{0}^{\infty} F_{n}(-\omega) \overline{\hat{\psi}}(-a \omega) e^{-\epsilon \omega} d \omega .
$$

By equations $111,(18)$ and $19,,\left(W_{\psi} f\right)$ has been obtained as follows

$$
\begin{aligned}
\left(W_{\psi} f\right)(b, a) & =\frac{a|a|^{-\frac{1}{2}}}{2 \pi} \sum_{k=0}^{n-1} d_{k} \times M[\overline{\hat{\psi}}(\omega) ; k+\sigma] \\
& +(-1)^{k+\sigma+1} M[\overline{\hat{\psi}}(-\omega) ; k+\sigma] \times a^{-k-\sigma} \\
& +\delta_{n}(a) ; \text { as } n \rightarrow+\infty
\end{aligned}
$$

where approximation or error bound $\delta_{n}$ is given by

$$
\delta_{n}(a)=\lim _{\epsilon \rightarrow 0^{+}} \int_{0}^{\infty} F_{n}(\omega) \overline{\hat{\psi}}(a \omega) e^{-\epsilon \omega} d \omega .
$$

The existence theorem for the formula ([1], theorem 6,p.217) has been obtained in the following form:

\subsection{Theorem}

If

(i) $\hat{f}^{(l)}(\omega)$ is continuous on $(-\infty, \infty)$,

(ii) for $0<\sigma \leq 1$, $\hat{f}(\omega)=\sum_{k=0}^{\infty} c_{k} \omega^{k+\sigma-1}$ as $\omega \rightarrow 0^{+}$;

(iii) $\overline{\hat{\psi}}(\omega) \sim e^{i \tau \omega^{p}} \sum_{r=0}^{\infty} b_{r} \omega^{-r-\alpha} ; \quad \alpha>0, \omega \rightarrow+\infty, \tau \neq$ $0, p \geq 1$

(iv) $\omega^{-\alpha} \hat{f}^{(j)}(\omega)=O\left(\omega^{-1-\epsilon}\right)$, as $\omega \rightarrow \infty$, for $j=0,1,2, \cdots, l$, $l$ being a non negative integer, $\epsilon>0$. 
then,

$$
\begin{aligned}
\left(W_{\psi} f\right)(b ; a) & =\frac{a|a|^{-\frac{1}{2}}}{2 \pi} \sum_{k=0}^{n-1} d_{k} \times M[\overline{\hat{\psi}}(\omega) ; k+\sigma] \\
& +(-1)^{k+\sigma+1} M[\overline{\hat{\psi}}(-\omega) ; k+\sigma] \times a^{-k-\sigma} \\
& +\delta_{n}(a) ; \text { as } n \rightarrow+\infty
\end{aligned}
$$

holds with approximation or error bound $\delta_{n}$ given by

$$
\delta_{n}(a)=\frac{(-1)^{l}}{a^{l}} \int_{-\infty}^{\infty} F_{n}^{(l)}(\omega)(\overline{\hat{\psi}}(a \omega))^{(-l)} d \omega
$$

and $\sigma+n>l$.

\subsection{Lemmas}

For the proof of the Theorem 3.1 following lemmas are required.

Lemma 1 If the integral $\int_{0}^{\infty} f(t) d t$ exists as an improper Riemann integral then,

$$
\lim _{\epsilon \rightarrow 0^{+}} \int_{0}^{\infty} e^{-(\epsilon-x) t} f(t) d t=\int_{0}^{\infty} f(t) d t
$$

(Wong [1], p. 197).

Lemma 2 For $x>0$ and $\operatorname{Re} \lambda>0$,

$$
\lim _{\epsilon \rightarrow 0^{+}} \int_{0}^{\infty} t^{\lambda-1} e^{-(\epsilon-i x) t} d t=\frac{e^{\lambda \pi i / 2} \Gamma(\lambda)}{x^{\lambda}},
$$

(Wong [1], p. 198).

Example 1 Let $f(t)$ be absolutely integrable on $[0, \delta]$ for some $\delta>0$, and be bounded for $t \geq \delta$. Then for any $p \geq 1$,

$$
\lim _{\epsilon \rightarrow 0^{+}} \epsilon \int_{0}^{\infty} f(t) e^{-\epsilon t^{p}} d t=0
$$

(Wong [1], p. 233).

\subsection{Proof of Theorem 3.1}

Integration by parts,

$$
\begin{aligned}
\int_{0}^{\infty} F_{n}(\omega) \overline{\hat{\psi}}(a \omega) e^{-\epsilon \omega^{p}} d \omega & =\left[\left(\int \overline{\hat{\psi}}(a \omega) d \omega\right) F_{n}(\omega) e^{-\epsilon \omega^{p}}\right]_{0}^{\infty} \\
& -\frac{1}{a} \int_{0}^{\infty} \overline{\hat{\psi}}^{(-1)}(a \omega) \\
& \times \frac{d}{d \omega} F_{n}(\omega) e^{-\epsilon \omega^{p}} d \omega .
\end{aligned}
$$

By condition $(i v)$ of Theorem 3.1 ,

$$
\left[\left(\int \overline{\hat{\psi}}(a \omega) d \omega\right) F_{n}(\omega) e^{-\epsilon \omega^{p}}\right]_{0}^{\infty}=0 .
$$

Then,

$$
\begin{aligned}
\int_{0}^{\infty} F_{n}(\omega) \overline{\hat{\psi}}(a \omega) e^{-\epsilon \omega^{p}} d \omega & =-\frac{1}{a} \int_{0}^{\infty} \overline{\hat{\psi}}^{(-1)}(a \omega) \\
& \times F_{n}^{(1)}(\omega) e^{-\epsilon \omega^{p}} d \omega \\
& +\frac{\epsilon p}{a} \int_{0}^{\infty} \overline{\hat{\psi}}^{(-1)}(a \omega) \\
& \times F_{n}(\omega) \omega^{p-1} e^{-\epsilon \omega^{p}} d \omega
\end{aligned}
$$

By Lemmas (1), (2) and example (1)

$$
\lim _{\epsilon \rightarrow 0^{+}} \frac{\epsilon p}{a} \int_{0}^{\infty} \overline{\hat{\psi}}^{(-1)}(a \omega) F_{n}(\omega) \omega^{p-1} e^{-\epsilon \omega^{p}} d \omega=0
$$

Thus,

$$
\delta_{n}^{(1)}=-\frac{1}{a} \lim _{\epsilon \rightarrow 0^{+}} \int_{0}^{\infty} \overline{\hat{\psi}}^{(-1)}(a \omega) F_{n}^{(1)}(\omega) e^{-\epsilon \omega^{p}} d \omega .
$$

Repeating this process $l$ times,

$$
\begin{aligned}
& \delta_{n}^{(1)}= \frac{(-1)^{l}}{(a)^{l}} \lim _{\epsilon \rightarrow 0^{+}} \int_{0}^{\infty} \overline{\hat{\psi}}^{(-l)}(a \omega) F_{n}^{(l)}(\omega) e^{-\epsilon \omega^{p}} d \omega \\
&= \frac{(-1)^{l}}{(a)^{l}} \int_{0}^{\infty} \overline{\hat{\psi}}^{(-l)}(a \omega) F_{n}^{(l)}(\omega) d \omega \\
& \text { by Lemma } 1 .
\end{aligned}
$$

Similarly

$$
\begin{aligned}
\int_{0}^{\infty} F_{n}(-\omega) \overline{\hat{\psi}}(-a \omega) e^{-\epsilon \omega^{p}} d \omega & =-\frac{1}{(a)} \int_{0}^{\infty} \overline{\hat{\psi}}^{(-1)}(-a \omega) \\
& \times \frac{d}{d \omega}\left(F_{n}(-\omega) e^{-\epsilon \omega^{p}}\right) d \omega . \\
& =\frac{1}{-a} \int_{0}^{\infty} \overline{\hat{\psi}}^{(-1)}(-a \omega) \\
& \times F_{n}^{(1)}(-\omega) e^{-\epsilon \omega^{p}} d \omega \\
& +\frac{\epsilon p}{a} \int_{0}^{\infty} \overline{\hat{\psi}}^{(-1)}(-a \omega) \\
& \times F_{n}(-\omega) \omega^{p-1} e^{-\epsilon \omega^{p}} d \omega \cdot(30)
\end{aligned}
$$

Since,

$$
\lim _{\epsilon \rightarrow 0^{+}} \frac{\epsilon p}{a} \int_{0}^{\infty} \overline{\hat{\psi}}^{(-1)}(a \omega) F_{n}(-\omega) \omega^{p-1} e^{-\epsilon \omega^{p}} d \omega=0,
$$

therefore

$$
\begin{aligned}
\delta_{n}^{(2)} & =\frac{(-1)^{l}}{(a)^{l}} \lim _{\epsilon \rightarrow 0^{+}} \int_{0}^{\infty} \overline{\hat{\psi}}^{(-l)}(-a \omega) F_{n}^{(l)}(-\omega) e^{-\epsilon \omega^{p}} d \omega \\
& =\frac{(-1)^{l}}{(a)^{l}} \int_{0}^{\infty} \overline{\hat{\psi}}^{(-l)}(-a \omega) F_{n}^{(l)}(-\omega) d \omega .
\end{aligned}
$$

Combining equations 29 and 31

$$
\begin{aligned}
\delta_{n}(a)= & \delta_{n}^{(1)}(a)+\delta_{n}^{(2)}(a) \\
= & \frac{(-1)^{l}}{(a)^{l}} \lim _{\epsilon \rightarrow 0^{+}} \int_{0}^{\infty} \overline{\hat{\psi}}^{(-l)}(a \omega) F_{n}^{(l)}(\omega) e^{-\epsilon \omega^{p}} d \omega \\
& +\frac{(-1)^{l}}{(a)^{l}} \lim _{\epsilon \rightarrow 0^{+}} \int_{0}^{\infty} \overline{\hat{\psi}}^{(-l)}(-a \omega) F_{n}^{(l)}(-\omega) e^{-\epsilon \omega^{p}} d \omega \\
= & \frac{(-1)^{l}}{(a)^{l}} \int_{0}^{\infty} \overline{\hat{\psi}}^{(-l)}(a \omega) F_{n}^{(l)}(\omega) d \omega \\
& +\frac{(-1)^{l}}{(a)^{l}} \int_{0}^{\infty} \overline{\hat{\psi}}^{(-l)}(-a \omega) F_{n}^{(l)}(-\omega) d \omega \\
= & \frac{(-1)^{l}}{(a)^{l}} \int_{-\infty}^{\infty} \overline{\hat{\psi}}^{(-l)}(a \omega) F_{n}^{(l)}(\omega) d \omega .
\end{aligned}
$$

Thus, Theorem 3.1 is completely established. 


\section{GENERALIZED MEXICAN HAT WAVELET}

In this section the approximation of asymptotic expansions for generalised Mexican hat wavelet has been determined in the following form

\subsection{Theorem}

If $\hat{f}(\omega)$ satisfies conditions of Theorem 3.1 then

$$
\begin{aligned}
\left(W_{\psi} f\right)(b, a) & =-\frac{a|a|^{-\frac{1}{2}}}{2 \pi} \sum_{k=0}^{n-1} d_{k} \frac{1}{2 \alpha^{\frac{k+\sigma+2}{2}}} \\
& \times \Gamma\left(\frac{k+\sigma+2}{2}\right)\left(1+(-1)^{k+\sigma-1}\right) a^{-k-\sigma} \\
& +\delta_{n}(a),
\end{aligned}
$$

holds with approximation

$$
\begin{aligned}
\delta_{n}(a) & =\frac{(-1)^{l+1}|a|^{-\frac{1}{2}}}{a^{l-1} 2 \pi} \int_{-\infty}^{\infty} F_{n}^{(l)}\left((a \omega)^{2} e^{-\alpha(a \omega)^{2}}\right)^{(-l)} d \omega \\
& =O\left(\int_{-\infty}^{\infty} F_{n}^{(l)}\left((a \omega)^{2} e^{-\alpha(a \omega)^{2}}\right)^{(-l)} d \omega .\right) .
\end{aligned}
$$

Proof:. The Gaussian function, denoted by $g_{\alpha}$, is defined by

$$
g_{\alpha}(t)=\frac{1}{2 \sqrt{\pi \alpha}} e^{-\frac{t^{2}}{4 \alpha}}, \quad \alpha>0,
$$

(Chui [6], pg. 50).

The generalized Mexican hat wavelet is defined by

$$
\psi(t)=-\frac{d^{2}}{d t^{2}}\left(g_{\alpha}(t)\right)=-\frac{1}{8 \alpha^{2} \sqrt{\pi \alpha}}\left(t^{2}-2 \alpha\right) e^{-\frac{t^{2}}{4 \alpha}} .
$$

The Fourier transform of Gaussian function is given as

$$
\hat{g_{\alpha}}(\omega)=e^{-\alpha \omega^{2}} .
$$

Using the well known result $\hat{f}^{(n)}(\omega)=(i \omega)^{n} \hat{f}(\omega)$,

$$
\begin{aligned}
\hat{\psi}(\omega) & =\omega^{2} e^{-\alpha \omega^{2}} \\
& =O\left(\omega^{2}\right), \text { as } \rightarrow 0^{+} .
\end{aligned}
$$

Since

$$
\hat{f}(\omega)=O\left(e^{\delta \omega^{2}}\right), \omega \rightarrow \infty \text { for some } \delta>0
$$

therefore,

$$
F(\omega)=e^{i b \omega} \hat{f}(\omega)=O\left(e^{\delta \omega^{2}}\right), \omega \rightarrow \infty .
$$

Thus,

$$
\begin{aligned}
\int_{0}^{\infty} \hat{f}(\omega) e^{i b \omega} \overline{\hat{\psi}}(a \omega) d \omega & =\sum_{k=0}^{n-1} d_{k} M\left[-\omega^{2} e^{-\alpha \omega^{2}} ; k+\sigma\right] a^{-k-\sigma} \\
& +\delta_{n}^{(1)}(a)
\end{aligned}
$$

where

$$
\begin{aligned}
M\left[\omega^{2} e^{-\alpha \omega^{2}} ; k+\sigma\right] & =\int_{0}^{\infty} \omega^{k+\sigma+1} e^{-\alpha \omega^{2}} d \omega \\
& =\frac{1}{2 \alpha^{\frac{k+\sigma+2}{2}}} \Gamma\left(\frac{k+\sigma+2}{2}\right)
\end{aligned}
$$

and

$$
\delta_{n}^{(1)}=\lim _{\epsilon \rightarrow 0^{+}} \int_{0}^{\infty} F_{n}(\omega)\left(-(a \omega)^{2} e^{-\alpha(a \omega)^{2}}\right) e^{-\epsilon \omega} d \omega .
$$

From equations 39 , and 40 ,

$$
\begin{aligned}
\int_{0}^{\infty} \hat{f}(\omega) e^{i b \omega} \overline{\hat{\psi}}(a \omega) d \omega & =\sum_{k=0}^{n-1} d_{k} \frac{1}{2 \alpha^{\frac{k+\sigma+2}{2}}} \Gamma\left(\frac{k+\sigma+2}{2}\right) \\
& \times a^{-k-\sigma}+\delta_{n}^{(1)}(a) .
\end{aligned}
$$

Similarly,

$$
\begin{aligned}
\int_{0}^{\infty} \hat{f}(-\omega) e^{-i b \omega} \overline{\hat{\psi}}(-a \omega) d \omega & =\sum_{k=0}^{n-1}(-1)^{k+\sigma-1} d_{k} \frac{1}{2 \alpha^{\frac{k+\sigma+2}{2}}} \\
& \times \Gamma\left(\frac{k+\sigma+2}{2}\right) a^{-k-\sigma}+\delta_{n}^{(2)}(a)
\end{aligned}
$$

where

$$
\delta_{n}^{(2)}=\lim _{\epsilon \rightarrow 0+} \int_{0}^{\infty} F_{n}(-\omega)\left(-(a \omega)^{2} e^{-\alpha(a \omega)^{2}}\right) e^{-\epsilon \omega} d \omega .
$$

By equations (1), 42, and 43,

$$
\begin{aligned}
\left(W_{\psi} f\right)(b, a) & =\frac{a|a|^{-\frac{1}{2}}}{2 \pi} \sum_{k=0}^{n-1} d_{k} \frac{1}{2 \alpha^{\frac{k+\sigma+2}{2}}} \\
& \times \Gamma\left(\frac{k+\sigma+2}{2}\right)\left(1+(-1)^{k+\sigma-1}\right) a^{-k-\sigma} \\
& +\delta_{n}(a)
\end{aligned}
$$

where

$$
\begin{aligned}
\delta_{n}(a) & =\delta_{n}^{(1)}(a)+\delta_{n}^{(2)}(a) \\
& =\lim _{\epsilon \rightarrow 0^{+}} \int_{0}^{\infty} F_{n}(\omega)(a \omega)^{2} e^{-\alpha(a \omega)^{2}} e^{-\epsilon \omega} d \omega \\
& +\lim _{\epsilon \rightarrow 0+} \int_{0}^{\infty} F_{n}(-\omega)(a \omega)^{2} e^{-\alpha(a \omega)^{2}} e^{-\epsilon \omega} d \omega \\
& \left.=\int_{0}^{\infty} F_{n}(\omega)(a \omega)^{2} e^{-\alpha(a \omega)^{2}}\right) d \omega \\
& +\int_{0}^{\infty} F_{n}(-\omega)(a \omega)^{2} e^{-\alpha(a \omega)^{2}} d \omega \\
& =\int_{-\infty}^{\infty} F_{n}(\omega)(a \omega)^{2} e^{-\alpha(a \omega)^{2}} d \omega .
\end{aligned}
$$

Considering above steps and the proof of the Theorem 3.1 the result has been completely established.

\section{THE WAVELET CORRESPONDING TO $M^{T H}$ ORDER CARDINAL $B-S P L I N E$}

In this section the approximation of asymptotic expansions for $m^{t h}$ Order Cardinal $B-$ spline has been determined in the following form: 


\subsection{Theorem}

If $\hat{f}(\omega)$ satisfies conditions of Theorem 3.1 then,

$$
\begin{aligned}
\left(W_{\psi} f\right)(b, a) & =\frac{|a|^{-\frac{1}{2}}}{2 \pi a^{m-1}} i^{m} g(b) \\
& +\frac{a|a|^{-\frac{1}{2}}}{2 \pi} \sum_{r=1}^{m} \sum_{k=1}^{n-1} i^{m+2 r} C_{r}^{m} d_{k}\left(1+(-1)^{k+\sigma+1}\right) \\
& \times e^{i \frac{k+\sigma-m}{2}} \Gamma(k+\sigma-m)(a r)^{-(k+\sigma-m)}+\delta_{n}(a),
\end{aligned}
$$

holds with approximation

$$
\begin{aligned}
\delta_{n}(a) & =\frac{i^{m}(-1)^{l}|a|^{-\frac{1}{2}}}{2 \pi a^{m+l}} \int_{-\infty}^{\infty} F_{n}^{(l)}(\omega)\left(\frac{e^{i r a \omega}}{(\omega)^{m}}\right)^{(-l)} d \omega \\
& =O\left(\int_{-\infty}^{\infty} F_{n}^{(l)}(\omega)\left(\frac{e^{i r a \omega}}{(\omega)^{m}}\right)^{(-l)} d \omega\right)
\end{aligned}
$$

where $n$ is the smallest positive integer such that $\sigma+n>l$. Proof: The $m^{t h}$ order cardinal B-spline

$$
\begin{aligned}
N_{m}(t)= & N_{1} * N_{1} * N_{1} * \ldots N_{1} \\
& \left(N_{1} \text { convolutes itself } m \text { times }\right) \\
= & \int_{0}^{1} N_{m-1}(t-x) d x, \quad m=2,3, \cdots .,
\end{aligned}
$$

where

$$
N_{1}(x)= \begin{cases}1, & x \in[0,1) \\ 0, & \text { otherwise }\end{cases}
$$

Chui ([б], p.56)

Then

$$
\begin{aligned}
\hat{\hat{N}}_{m}(\omega) & =\left(\frac{1-e^{-i \omega}}{i \omega}\right)^{m} \\
& =\left(\frac{i}{\omega}\right)^{m}\left(1-e^{i \omega}\right)^{m} \\
& =\left(\frac{i}{\omega}\right)^{m} \sum_{r=0}^{m}(-1)^{r} C_{r}^{m} e^{i \omega r} \\
& =\left(\frac{i}{\omega}\right)^{m}+(i)^{m} \sum_{r=1}^{m}(-1)^{r} C_{r}^{m} \frac{e^{i \omega r}}{\omega^{m}} \\
& =\left(\frac{i}{\omega}\right)^{m}+\sum_{r=1}^{m} i^{m+2 r} C_{r}^{m} \frac{e^{i \omega r}}{\omega^{m}} .
\end{aligned}
$$

Since,

$$
\lim _{\omega \rightarrow 0^{+}} \hat{\hat{N}}_{m}(\omega)=\lim _{\omega \rightarrow 0^{+}} e^{i m \frac{\omega}{2}}\left(\frac{\sin \frac{\omega}{2}}{\frac{\omega}{2}}\right)^{m}=1,
$$

therefore

$$
\hat{N}_{m}(\omega)=O(1) \quad \text { as } \quad \omega \rightarrow 0^{+} .
$$

By equations.(6), 2) and (49),

$$
\left(W_{\psi} f\right)(b, a)=\frac{a|a|^{-\frac{1}{2}}}{2 \pi} \int_{-\infty}^{\infty} \hat{f}(\omega) e^{i b \omega}
$$

$$
\begin{aligned}
& \times\left(\left(\frac{i}{a \omega}\right)^{m}+\sum_{r=1}^{m} i^{m+2 r} C_{r}^{m} \frac{e^{i a \omega r}}{a^{m} \omega^{m}}\right) d \omega \\
& =\frac{|a|^{-\frac{1}{2}}}{2 \pi a^{m-1}} i^{m} \int_{-\infty}^{\infty} \frac{\hat{f}(\omega) e^{i b \omega}}{\omega^{m}} d \omega \\
& +\frac{a|a|^{-\frac{1}{2}}}{2 \pi} \sum_{r=1}^{m} i^{m+2 r} C_{r}^{m} \int_{-\infty}^{\infty} \hat{f}(\omega) e^{i b \omega} \frac{e^{i a \omega r}}{a^{m} \omega^{m}} d \omega \\
& =\frac{|a|^{-\frac{1}{2}}}{2 \pi a^{m-1}} i^{m} g(b)+\frac{a|a|^{-\frac{1}{2}}}{2 \pi} \sum_{r=1}^{m} i^{m+2 r} C_{r}^{m} \\
& \times \int_{0}^{\infty} \hat{f}(\omega) e^{i b \omega} \frac{e^{i a r \omega}}{a^{m} \omega^{m}} d \omega \\
& +\int_{0}^{\infty} \hat{f}(-\omega) e^{-i b \omega} \frac{e^{-a i \omega r}}{(-a)^{m} \omega^{m}} d \omega,
\end{aligned}
$$

where

$$
g(b)=\int_{-\infty}^{\infty} \frac{\hat{f}(\omega) e^{i b \omega}}{\omega^{m}} d \omega .
$$

The generalized Mellin transform formula of $e^{i t}$ is given by

$$
\begin{aligned}
M\left[e^{i t} ; z\right] & =\lim _{\epsilon \rightarrow 0^{+}} \int_{0}^{\infty} t^{z-1} e^{i t} e^{-\epsilon t} d t \\
& =\lim _{\epsilon \rightarrow 0^{+}} \int_{0}^{\infty} t^{z-1} e^{-t(\epsilon-i)} d t \\
& =\lim _{\epsilon \rightarrow 0^{+}} \int_{0}^{\infty}\left(\frac{x}{\epsilon-i}\right)^{z-1} e^{-x} \frac{1}{(\epsilon-i)} d x \\
& =\lim _{\epsilon \rightarrow 0^{+}} \frac{1}{(\epsilon-i)^{z}} \int_{0}^{\infty} x^{z-1} e^{-x} d x \\
& =\lim _{\epsilon \rightarrow 0^{+}} \frac{1}{(\epsilon-i)^{z}} \Gamma z \\
& =\frac{1}{(-i)^{z}} \Gamma z \\
& =e^{i \pi \frac{z}{2}} \Gamma z, i=e^{\frac{i \pi}{2}} .
\end{aligned}
$$

By (Wong [1], p.192,)

$$
M\left[\frac{e^{i r \omega}}{\omega^{m}} ; k+\sigma\right]=e^{i \frac{\pi}{2}(k+\sigma-m)} \Gamma(k+\sigma-m) r^{-(k+\sigma-m)} .
$$

By equation (7),

$$
\begin{aligned}
\int_{0}^{\infty} \hat{f}(\omega) e^{i b \omega}\left(\frac{e^{i a \omega r}}{a \omega^{m}}\right) & =\sum_{k=0}^{n-1} d_{k} M\left[\frac{e^{i a \omega r}}{a^{m} \omega^{m}} ; k+\sigma-m\right] \\
& \times a^{-(k+\sigma-m)} \\
& +\lim _{\epsilon \rightarrow 0^{+}} \int_{0}^{\infty} F_{n}(\omega) \frac{e^{i a \omega r}}{a^{m} \omega^{m}} e^{-\epsilon \omega} d \omega \\
& =\sum_{k=0}^{n-1} d_{k} e^{i \frac{(k+\sigma-m)}{2}} \\
& \times \Gamma(k+\sigma-m)(a r)^{-(k+\sigma-m)} \\
& +\lim _{\epsilon \rightarrow 0^{+}} \int_{0}^{\infty} F_{n}(\omega) \frac{e^{i a \omega r}}{a^{m} \omega^{m}} e^{-\epsilon \omega} d \omega
\end{aligned}
$$


Similarly,

$$
\begin{aligned}
\int_{0}^{\infty} \hat{f}(-\omega) e^{-i b \omega}\left(\frac{e^{-i a \omega r}}{(-a)^{m} \omega^{m}}\right) & =\sum_{k=0}^{n-1}(-1)^{k+\sigma+1} d_{k} e^{i \frac{(k+\sigma-m)}{2}} \\
& \times \Gamma(k+\sigma-m)(a r)^{-(k+\sigma-m)} \\
& +\lim _{\epsilon \rightarrow 0^{+}} \int_{0}^{\infty} F_{n}(-\omega) \\
& \times \frac{e^{-i a \omega r}}{(-a)^{m} \omega^{m}} e^{-\epsilon \omega} d \omega
\end{aligned}
$$

By equations 51, 54, and 55,

$$
\begin{aligned}
\left(W_{\psi} f\right)(b, a) & =\frac{|a|^{-\frac{1}{2}}}{2 \pi a^{m-1}} i^{m} g(b) \\
& +\frac{a|a|^{-\frac{1}{2}}}{2 \pi} \sum_{r=1}^{m} \sum_{k=1}^{n-1} i^{m+2 r} C_{r}^{m} d_{k}\left(1+(-1)^{k+\sigma+1}\right) \\
& \times e^{i \frac{k+\sigma-m}{2}} \Gamma(k+\sigma-m)(a r)^{-(k+\sigma-m)} \\
& +\delta_{n}(a)
\end{aligned}
$$

where

$$
\begin{aligned}
\delta_{n}(a) & =\lim _{\epsilon \rightarrow 0^{+}} \int_{0}^{\infty} F_{n}(\omega) \frac{e^{i a \omega r}}{a^{m} \omega^{m}} e^{-\epsilon \omega} d \omega \\
& +\lim _{\epsilon \rightarrow 0^{+}} \int_{0}^{\infty} F_{n}(-\omega) \frac{e^{-i a \omega r}}{(-a)^{m} \omega^{m}} e^{-\epsilon \omega} d \omega \\
& =\int_{0}^{\infty} F_{n}(\omega) \frac{e^{i a \omega r}}{a^{m} \omega^{m}} d \omega+\int_{0}^{\infty} F_{n}(-\omega) \frac{e^{-i \bar{a} \omega r}}{-a^{m} \omega^{m}} d \omega \\
& =\int_{-\infty}^{\infty} F_{n}(\omega)\left(\frac{e^{i a \omega r}}{a^{m} \omega^{m}} d \omega\right) .
\end{aligned}
$$

By the help of above mentioned steps and the proof of the Theorem 3.1, this result has been proved.

\section{CONCLUSION}

(i) Estimates for the asymptotic expansion of generalized Mexican hat wavelet and $m^{\text {th }}$ order cardinal $B$-spline have been obtained.

(ii) The result of Pathak and Pathak ([3] Theorem(4)) is a particular case of Theorem 4.1 in this paper if $\alpha=\frac{1}{2}$.

(iii) If $m=2$, then estimate for the asymptotic expansion of $2^{\text {nd }}$ order cardinal $B$-spline satisfies

$$
\delta_{n}(a)=O\left(\int_{-\infty}^{\infty} F_{n}^{(l)}(\omega) e^{-i r a l \omega} \omega^{m l} d \omega\right),
$$

where $n$ is the smallest positive integer such that $\sigma+n>l$. This estimate may be developed independently as similar to Theorem 5.1 taking $\mathrm{m}=2$. (iv) For $m=1, m^{\text {th }}$ order $B$-spline reduced to

$$
N_{1}(x)=\left\{\begin{array}{lc}
1, & x \in[0,1) ; \\
0, & \text { otherwise },
\end{array}\right.
$$

This is the first order $B-$ spline.

$$
\begin{gathered}
\hat{N}_{1}(\omega)=e^{-i \frac{\omega}{2}} \frac{\sin \frac{\omega}{2}}{\frac{\omega}{2}} \\
\hat{N}_{1}(0)=\lim _{\omega \rightarrow 0}\left(e^{-i \frac{\omega}{2}} \frac{\sin \frac{\omega}{2}}{\frac{\omega}{2}}\right) \\
=1
\end{gathered}
$$

$N_{1}(t)$ is a scaling function. It is not a wavelet.The Theorem 5.1 is not applicable. Thus, the estimate for $m^{t h}$ order $B$-spline has been obtained for $m \geq 2$.

\section{ACKNOWLEDGMENTS}

Shyam Lal, one of the authors, is thankful to DST - CIMS for encouragement to this work.

Susheel Kumar, one of the authors, is grateful to C.S.I.R. (India) in the form of Junior Research Fellowship vide Ref. No. 19-06/2011 (i)EU-IV Dated:03-10-2011 for this research work.

Authors are grateful to the referee for his valuable comments and suggestions for the improvement of this paper.

\section{REFERENCES}

[1] Wong, R., Asymptotic Approximations of Integrals, Academic Press, New York (1989).

[2] W. Sweldens and R. Pensiess, Quadrature formulae and asymptotic error expansions for wavelet approximation, of smooth function,Siam J. Numei. Anal.Vol. 31, No. 4, pp. 12401264, August 1994.

[3] R.S.Pathak and A. Pathak, Asymptotic Expansion of the Wavelet transform with error term World Scientific(2009),ISBN:978-90-78677-26-0, pp:154-164.

[4] Wong, R., Explicit error terms for asymptotic expansions of Mellin convolutions, J. Math. Anal. Appl. 72(1979), 740-756.

[5] R. Ashurov and A. Butaev, On continuous wavelet transforms of distributions,Applied Mathematics Letters 24 (2011) 15781583.

[6] Chui C. K., An introduction to wavelets (Wavelet analysis and its applications), Vol. 1, Academic Press, USA, (1992). 